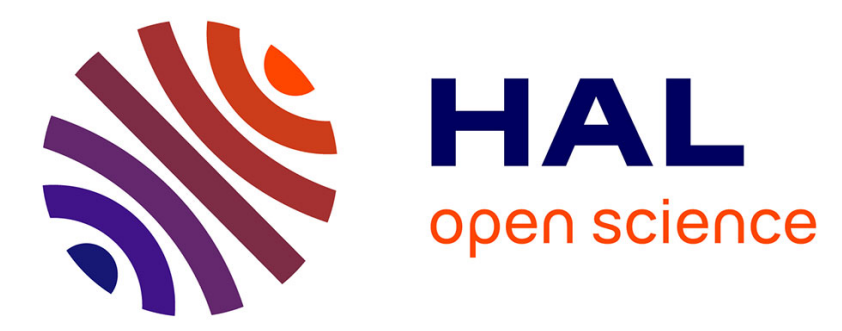

\title{
Triterpene saponins from Fagonia scabra Forssk and other Fagonia species
}

Naima Boutaghane, Zahia Kabouche, Laurence Voutquenne-Nazabadioko

\section{To cite this version:}

Naima Boutaghane, Zahia Kabouche, Laurence Voutquenne-Nazabadioko. Triterpene saponins from Fagonia scabra Forssk and other Fagonia species. Biochemical Systematics and Ecology, 2016, 67, pp.1-6. 10.1016/j.bse.2016.05.017 . hal-01996485

\section{HAL Id: hal-01996485 \\ https://hal.univ-reims.fr/hal-01996485}

Submitted on 28 Oct 2021

HAL is a multi-disciplinary open access archive for the deposit and dissemination of scientific research documents, whether they are published or not. The documents may come from teaching and research institutions in France or abroad, or from public or private research centers.
L'archive ouverte pluridisciplinaire HAL, est destinée au dépôt et à la diffusion de documents scientifiques de niveau recherche, publiés ou non, émanant des établissements d'enseignement et de recherche français ou étrangers, des laboratoires publics ou privés. 
Triterpene saponins from Fagonia scabra Forssk and other Fagonia species.

Naima Boutaghane, ${ }^{\mathrm{a}, \mathrm{b}}$ Zahia Kabouche ${ }^{\mathrm{b}}$, Laurence Voutquenne -Nazabadioko*a

anstitut de Chimie Moléculaire de Reims (ICMR), CNRS UMR 7312, UFR de Pharmacie, BP 1039, 51687 Reims; France

${ }^{b}$ Université des frères Mentouri-Constantine, département de chimie, Laboratoire d'Obtention de Substances Thérapeutiques (LOST), Campus Chaabet Ersas, 25000 Constantine, Algeria

\footnotetext{
* Email: laurence.nazabadioko@univ-reims.fr
} 


\section{Highlights}

- Eight compounds were isolated from Fagonia scabra for the first time

- Six compounds were isolated from genus Fagonia for the first time

- Luteolin-7-O- $\beta$-D-glucopyranoside was a chemotaxonomic marker of $F$. scabra

- It is the first luteolin flavonoid isolated from Zygophyllaceae family

- Saponins from F. scabra were compared with other saponins from Fagonia species. 


\begin{abstract}
This article reports on the structure of triterpenoids saponins (1-49) isolated from Fagonia species. In addition, it describes one known flavonoid (50) with luteolin as aglycone and seven known triterpenoid saponins $(\mathbf{2 6}, \mathbf{5 1 - 5 6})$ isolated from the aerial parts of Fagonia scabra Forssk. Compounds 50-56, were isolated for the first time from the genus Fagonia, while compounds 50-53, 55-56 have never been encountered in the Zygophyllaceae family. The chemotaxonomic relationship between $F$. scabra and other Fagonia species was also discussed.
\end{abstract}

Keywords : Fagonia scabra, Fagonia microphylla, Zygophyllaceae, Triterpenoid saponins, luteolin 


\section{Subject and source}

The Zygophyllaceae family is composed of approximately 27 genera and 285 species, mostly distributed in hyperarid and arid zones of the old and new world (Engler, 1931; Sheahan and Chase, 1996; Beier, 2003). Among these, Fagonia is one of the main genera with 34 desert plant species (Beier, 2003; Beier et al., 2004), and is represented by 17 species in the flora of Algeria (Quezel and Santa, 1963). Several of these are used in folk medicine, for their antiinflammatory activity, analgesic and anti-pyretic effects, thrombolytic effects and antioxidant activity (Chopra et al., 1982; Saeed Asif and Sabir Wahid, 2003; Kasture et al., 2014). They are also used as popular remedies for the treatment of various skin lesions, and digestive disorders (Chopra et al., 1982).

Aerial parts of Fagonia scabra Forssk (= F. microphylla Pomel as synonym) were collected near Ghardaia (Algerian Septentrional Sahara) in April 2009. The botanical identification was made by Pr. Gerard De Bélair (University of Annaba, Algeria). A voucher specimen (ZKFm04/09) was deposited in the Herbarium of the Biology Department (Université Mentouri-Constantine, Algeria).

\section{Previous work}

Previous phytochemical studies on Fagonia species demonstrated the presence of flavonoids (Saleh et al., 1990; Al-Wakeel 1992; El-Wakil and Eman, 2007), diterpenoids (Abdel-Kader et al., 1993; Perrone et al. 2007; Sallam et al., 2014), sterols glycosides (Shoeb et al. 1994), triterpenes (Anjum et al., 2007; Saleem et al., 2014; Farheen et al., 2015) and saponins (Ansari et al., 1988; Miyase et al., 1996a; Melek et al., 2000; Batterjee et al., 2002; Sallam et al., 2014). A total of forty-nine triterpenoid saponins (1-49) have been identified in the five investigated Fagonia species, F. arabica L. (Miyase et al., 1996a; Shoeb et al, 1994; Perrone et al., 2007), F. cretica L. (Abdel Khalik et al., 2000a,b; Batterjee et al., 2002; Anjum et al., 2014; Saleem et al., 2014), F. glutinosa Delile (Melek et al., 2000), F. indica Burm.f. (Ansari et al., 1987, 
1988; Shaker et al., 1999, 2000, 2013; Farheen et al., 2015), and F. mollis Delile (Sallam et al., 2014). Their structures are reported in Table 1 and Fig. 1.

\section{Present study}

Powdered air-dried aerial parts of $F$. scabra $(1 \mathrm{~kg})$ were extracted by petroleum ether $(15 \mathrm{~L})$. The defatted powder was then macerated in $\mathrm{MeOH}-\mathrm{H}_{2} \mathrm{O}(8: 2,12 \mathrm{~L})$ for $24 \mathrm{~h}$ and refluxed for 3 $\mathrm{h}$ to afford $89.3 \mathrm{~g}$ of a dark residue after filtration and solvent evaporation. The residue was dissolved in $\mathrm{MeOH}(600 \mathrm{ml})$ and precipitated by addition of a large excess of $\mathrm{Me}_{2} \mathrm{CO}(3 \mathrm{~L})$. The resulting precipitate was filtered off, dried over $\mathrm{KOH}$ and dissolved in $\mathrm{H}_{2} \mathrm{O}$ and dialyzed against pure distilled $\mathrm{H}_{2} \mathrm{O}$ in seamless cellulose tubing (Spectra/por dialysis membrane, MCWO 6-8.0000, Spectum lab, France) under stirring during $48 \mathrm{~h}$. The content of the tubes was freeze-dried to afford $3.6 \mathrm{~g}$ of a saponin mixture. This crude mixture was subjected to vacuum liquid chromatography over $\mathrm{RP}-18$ using a gradient of $\mathrm{MeOH}-\mathrm{H}_{2} \mathrm{O}(4: 6,6: 4,8: 2$ and 0:1, each $250 \mathrm{ml}$ ) to give four fractions [ $\left.\mathrm{F}_{1}: 0.93 \mathrm{~g}, \mathrm{~F}_{2}: 1.19 \mathrm{~g}, \mathrm{~F}_{3}: 1.3 \mathrm{~g}, \mathrm{~F}_{4}: 0.16 \mathrm{~g}\right]$. Fraction $\mathrm{F}_{3}(1.3 \mathrm{~g})$ was purified by silica gel $\mathrm{CC}$, eluted with a $\mathrm{CHCl}_{3}-\mathrm{MeOH}-\mathrm{H}_{2} \mathrm{O}$ gradient mixture [95:5:0-70:30:5], to afford pure compound $\mathbf{5 0}(600 \mathrm{mg})$ as a yellow precipitate. Fractions [3646] (451 mg), eluted with $\mathrm{CHCl}_{3}-\mathrm{MeOH}$ (9:1), were purified by semi prep HPLC using an isocratic program of $44 \% \mathrm{MeCN}$ in $\mathrm{H}_{2} \mathrm{O}$ during $10 \mathrm{~min}$ to yield $\mathbf{5 1}$ (10 mg). Fractions [47-89] (334 mg), eluted with $\mathrm{CHCl}_{3}-\mathrm{MeOH}$ (8:2), were purified by semi prep HPLC using an isocratic eluent of 36\% MeCN in $\mathrm{H}_{2} \mathrm{O}$ during $20 \mathrm{~min}$ to give $\mathbf{5 2}$ (17.2 mg), 53 (9.6 mg), 54 (50 mg), and 51 (15 mg). Saponin 55 (15 mg) was purified from fractions [90-120], eluted with $\mathrm{CHCl}_{3-}$ $\mathrm{MeOH}$ (7:3), by semi-prep HPLC using a linear gradient from $36 \%$ to $42 \% \mathrm{MeCN}$ in $\mathrm{H}_{2} \mathrm{O}$ during 15 min. Fraction $\mathrm{F}_{4}(0.16 \mathrm{~g})$ was subjected to silica gel $\mathrm{CC}$ using a gradient of $\mathrm{CHCl}_{3-}$ $\mathrm{MeOH}-\mathrm{H}_{2} \mathrm{O}$ [9:1:0 to $\left.12: 8: 1\right]$. Fractions [39-49] (17 mg) eluted with $\mathrm{CHCl}_{3}-\mathrm{MeOH}(85: 15)$ were purified by RP-18 CC, using a gradient of $\mathrm{MeOH}-\mathrm{H}_{2} \mathrm{O}$ [4:6 to 6:4] to give $26(6 \mathrm{mg})$ and $56(2.2 \mathrm{mg})$. 
The structures of the isolated compounds were determined by 1D and 2D NMR experiments, and by comparison with data from the literature. Compound $\mathbf{5 0}$ was identified as luteolin-7-O$\beta$-D-glucopyranoside (Agrawal, 1989), and was the major compound of the hydromethanolic extract. Other compounds were chikusetsaponin IVa (51) (Lin et al., 1976), spinasaponin A 28$O$ - $\beta$-D-glucopyranoside (52) (Paphassarang et al., 1989), pseudoginsenoside RT1 (53) (Tanaka et al., 1985), quinosid D (54) (Mizui et al., 1990), 3-O- $\beta$-D-xylopyranosyl-(1 $\rightarrow 3)-[\beta$-Dglucopyranosyl-( $1 \rightarrow 2)]$ - $\beta$-D-glucuronopyranosyl-28- $O$ - $\beta$-D-glucopyranosyl-oleanolic $\quad$ acid (55) (Ridout et al., 1994), matesaponin 1 (26) (Miyase et al., 1996b), and matesaponin B (56) (Sachiko et al., 2009 ) (Fig. 2).

\section{Chemotaxonomic significance}

The present study reports the isolation and structural elucidation of one flavonoid (50) and of seven triterpenoid saponins $(\mathbf{2 6}, \mathbf{5 1 - 5 6})$ from the hydromethanolic extract of the defatted aerial parts of F. scabra (Fig. 2). Compounds 50-56, were isolated for the first time from the genus Fagonia. Compounds 50-53 and $\mathbf{5 5}$ have not been reported from any species in Zygophyllaceae family.

Triterpenoid saponins are bitter compounds frequently present in plants from arid countries to deter predation. They are representative secondary metabolites of Fagonia species and 49 of them (Fig 1) have been identified from five Fagonia species (Table 1). These saponins were mono-or disaccharides of oleanane triterpenoid, such as oleanolic acid $(\mathbf{1}, \mathbf{1 3 - 1 9}, \mathbf{3 6}, \mathbf{4 1})$, hederagenin (2, 33-35), and 27-hydroxyoleanolic acid (3-4, 20-21, 37-38), or ursane triterpenoid, such as ursolic acid (24-30, 43-44), 27-hydroxyursolic acid (22-23, 31-32), and quinovic acid $(6-7,10-12,42)$. The carbonyl in C-28 position was free or esterified by a $\beta$-Dglucopyranosyl unit or by a gentiobiosyl unit $(\beta$-D-glucopyranosyl-( $1 \rightarrow 6)-\beta$-D-glucopyranosyl)

(Fig.1). The hydroxyl in C-3 position was also glycosylated by a $\beta$-D-glucopyranosyl unit (2, 6-7, 10, 45-47) or by an $\alpha$-L-arabinopyranosyl which can be substituted in C-2 and/or C-3 
positions by a $\beta$-D-glucopyranosyl and/or a $\beta$-D-xylopyranosyl units (11-32, 35-40, 43-44). Saponins isolated from Fagonia cretica (Batterjee et al., 2002) were slightly different with an $\alpha$-L-rhamnopyranosyl substituted in C-4 position by a $\beta$-D-glucopyranosyl unit (33) or a disaccharide moiety $(\beta$-D-glucopyranosyl-( $(\rightarrow 5)$ - $\alpha$-L-arabinofuranosyl) $(\mathbf{3 4})$. In fagonoside A (49) isolated from $F$. cretica (Anjum et al., 2014) a 6-deoxy- $\alpha$-L-talopyranosyl was attached to

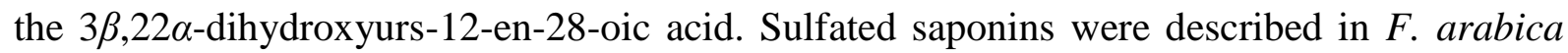
(Perrone et al. 2007) (3-4, 8-9, 22-23), F. indica (Shaker et al., 1999) (43-44), and F. mollis with quinovic acid (7) (Sallam et al., 2014). On the other hand three taraxastane saponins were characterized in F. indica (45-46) (Ansari et al., 1987; Farheen et al., 2015) with fagonicin or 3 $\beta, 20 S$-dihydroxytaraxastane-28-al and two nahagenin derivatives were isolated from $F$. arabica (8) (Perrone et al. 2007) and F. indica (48) (Ansari et al., 1987).

Our investigation of $F$. scabra adds to this list five oleanane (51-55), and two ursane (26, 56). triterpenoid saponins. Matesaponin 1 (26) with ursolic acid as aglycon was previously isolated from F. indica (Shaker et al., 2013) and Larrea tridentate (Sesse. \& Moc. Ex DC.) Coville (Zygophyllaceae) (Jitsuno and Mimaki, 2010). Compound 56 is a 23-hydroxyursolic acid glycoside isolated from Ilex paraguariensis St. Hilaire (Aquifoliaceae) (Sachiko et al., 2009) and was isolated for the first time from a Fagonia species. This saccharide moiety is also present in matesaponin 1 (26) with an $\alpha$-L-arabinopyranosyl attached to the position C-3 of the aglycone, which is a characteristic of this genus Fagonia. Except for quinosid D (54) previously isolated from Larrea tridentate (Zygophyllaceae) (Jitsuno and Mimaki, 2010), all others saponins (51-53 and 55) were isolated for the first time in Zygophyllaceae. Compound $\mathbf{5 1}$ have been isolated from Panax japonica (=P. japonicus (T. Ness) C.A.Mey) (Araliaceae) (Lin et al., 1976) and Beta vulgaris L. (Chenopodiaceae) (Yoshikawa et al., 1996). Compounds 52 and 53 have been isolated from Panax species (Araliaceae) (Paphassarang et al., 1989), whereas compound 55 was isolated from Beta vulgaris (Chenopodiaceae) (Ridout et al., 1994) for 
example. Compounds 51-55 were glycosides of oleanolic acid as in compounds 1, 13-19, 36, and 41, but differ by their saccharide moieties. A $\beta$-D-glucuronopyranosyl unit, substituted in position C-2 and/or C-3 by $\beta$-D-glucopyranosyl and/or $\beta$-D-xylopyranosyl units, was attached to the hydroxyl in position $\mathrm{C}-3$ of the aglycone (Fig.2). This significant chemical difference indicates a different chemotaxonomic feature and may contribute to enhancing our understanding of the taxonomy and evolution of the genus Fagonia.

In addition to saponins, the Fagonia species showed remarkably a homogeneous flavonoid profile in which quercetin, kaempferol, isorhamnetin, herbacetin, and herbacetin 8-methyl ether and their glycosides are predominant (Saleh et al., 1990; Abdel-Kader et al., 1993; El-Wakil and Eman, 2007). Compound 50, luteolin-7- $O-\beta$-D-glucopyranoside, has been reported to occur in many plants (Agrawal, 1989). To our knowledge, this is the first flavonoid with luteolin as aglycone isolated from Fagonia genus and Zygophyllaceae family (Saleh et al., 1990; AlWakeel, 1992).

In summary, this study extends the knowledge of the metabolites of $F$. scabra, suggesting that they might be regarded as chemotaxonomic markers. F. scabra is endemic to the Saharo-Sind region and Western Moroccan as well as F. glutinosa, F. arabica and F. cretica but they are not in the same clade (Beier et al., 2004), which is confirmed by the presence of quite a different secondary metabolism.

\section{Acknowledgments}

The authors wish to express their thanks to the Algerian Ministry of Higher Education and Research (MESRS) MESRS for financial support and CNRS, Conseil Regional Champagne Ardenne, Conseil General de la Marne, and EU-programme FEDER to the PIANET CPER project is gratefully acknowledged. We are also very grateful to Dr. G. Massiot (ICMR, CNRS UMR 7312) for critical reading of the manuscript.

\section{References}

Abdel-Kader, M.S., Omar, A.A., Abdel-Salam, N.A., Stermitz, F.R., 1993. Phytochemistry 33, 
718.

Abdel-Khalik, S.M., Miyase, T., El-Ashaal, H., Melek, F.R., 2000a. Phytochemistry 54, 853.

Abdel-Khalik, S.M., Miyase, T., Melek, F.R., El-Ashaal, HA., 2000b. Pharmazie 56, 247.

Agrawal, P.K., 1989. Central Institute of Medical and Aromatic Plants, India. Edition Elsevier, New York.

Al-Wakeel, S.A.M., 1992. Biochem. Syst. Ecol. 20, 259.

Anjum, M.I., Ahmed, E., Sharif, A., Jabbar, A., Malik, A., Hussain, T., Farooqi, Z.H., Nawaz, A.A., 2014. Asian J. Chem. 26, 7386.

Ansari, A., Kenne, L., Atta-ur-Rahman., 1987. Phytochemistry 26, 1487.

Ansari, A., Kenne, L., Atta-ur-Rahman., Wehler, T., 1988. Phytochemistry 27, 3979.

Batterjee, S.M., Shoeb, H., Refahy, L.A., El-Hashash, M.A., 2002. Int. J. Chem. 12, 143.

Beier, B.A., 2003. Acta Univ. Ups, Uppsala: 2

Beier, BA., Nylander, J.A.A., Chase, M.W., Thulin, M., 2004. Mol. Phylogenet. 33, 91.

Chopra, RM., Handa, K.L., Kapur, L.D., Chopra, I.C., 1982. Indigenous Drugs of India, second edition. Academic Press, New Delhi, India, 507.

El-Wakil, E., Eman A., 2007. Z. Naturforsch. C: J. BioSc. 62, 661.

Engler, A., 1931. In: Die Naturlichen Pflanzen familien, 2nd edition; Engler, A, Prantl, K, Eds, Engelimann: Leipzig 19a, 144.

Farheen, R., Shaheen, S.B., Mahmood, I., Shabana, S., Majeed, S., 2015. Phytochem. Let. 13, 256.

Jitsuno, M., Mimaki, Y., 2010. Phytochemistry 71, 2157.

Kasture, V.S., Gosavi, S.A., Kolpe, J.B., Deshapand, S.G., 2014. J. Pharm. Sc. 3, 1206.

Lin, T.D., Kondo, N., Shoji, J., 1976. Chem. Pharm. Bull. 24, 253.

Melek, F.R., Miyase, T., El-Gindy, M.R., Abdel Khalik, S.M., Ghaly, N.S., El-Kady, M., 2000.

Pharmazie 55, 772. 
Miyase,T., Melek, F.R., El-Gindi, O.D., Abdel-Khalik, S.M., El-Gindi, M.R, Haggag, M.Y.,1996a. Phytochemistry 41, 1175.

Miyase, T., Shiokawa, K.I., Zhang, D.M., Ueno, A., 1996b. Phytochemistry 41, 1411.

Mizui, F., Kasai, R., Ohtani, K., Tanaka, O., 1990. Chem. Pharm. Bull. 38, 375.

Paphassarang, S., Raynaud, J., Lussignol, M., Becchi, M., 1989. Phytochemistry 28, 1541.

Perrone, A., Masullo, M., Bassarello, C., Hamed, A., Belisario, AM., Pizza, C., Piacente, S., 2007. J. Nat. Prod. 70, 584.

Quezel, P., Santa, S., 1963. Nouvelle flore de l'Algérie et des régions désertiques méridionales. Tome I, CNRS, Paris. 588.

Ridout, C.L., Price, K.R., Parkin, G., Dijoux, M.G., Lavaud, C., 1994. J. Agric. Food Chem. 42, 279.

Sachiko, S., Seikou, N., Sachiyo, Y., Chihiro, Y., Yoshimi, O., Hisashi, M., Masayuki, Y., 2009. Chem. Pharm. Bull. 57, 257.

Saeed Asif, M., Sabir Wahid, A., 2003. J. Ethnopharmacol. 85, 195.

Saleem, S., Jafri, L., Haq, I., Chang, L.C., Calderwood, D., Green, B.D., Mirza, B., 2014. J. Ethnopharmacol. 156, 26.

Saleh, N.A., El-Hadidi, M.N., Al-Wakeel, S.A., 1990. Biochem. Syst. Ecol. 18, 49.

Sallam, A., Eko Nugroho, A., Hirasawa, Y., Chin-Piow, W., Kaneda,T., Shirota, O., Gedarab, S.R., Moritaa, H., 2014. Nat. Prod. Commun. 9, 1243.

Shaker, K.H., Bernhardt, M., Elgamal, M.H., Seifert, K., 1999. Phytochemistry 51, 1094.

Shaker, K.H., Bernhardt, M., Elgamal, M.H., Seifert, K., 2000. Z. Naturforsch. 55, 520.

Shaker, K.H., Al Jubiri, S.M., Abd El-hady, F.K., Al-Sehemi, A.G., 2013. Int. J. Pharmacol. Sci. 23, 231.

Sheahan, M.C., Chase, M.W., 1996. Bot. J. Linn. Soc. 122, 279.

Shoeb, H.A., Sharada, H., El-Sayed, M.M., Refahy, L.A., El-Waheel, E., 1994. J. Pharm. Sc. 
$13,41$.

Tanaka, O., Morita T., Kasai, R., Kinouchi, J., Sanada, S., Ida, Y., Shoji, J., 1985. Chem. Pharm. Bull. 33, 2323.

Yoshikawa, M., Murakami, T., Kadoya, M., Matsuda, H., Muraoka, O., Yamahara, J., Murakami, N., 1996. Chem. Pharm. Bull. 44, 1212. 
Table 1.Triterpenoid saponins of Fagonia species

\begin{tabular}{|c|c|c|c|}
\hline Fagonia sp & Triterpenoid saponin & $\mathbf{N}^{\circ}$ & Reference \\
\hline \multirow[t]{17}{*}{ F. arabica } & 3-O- $\beta$-D-glucopyranosyl-oleanolic acid & 1 & Shoeb et al. 1994 \\
\hline & 3- $O$ - $\alpha$-L-arabinopyranosyl-quinovic acid-28- $O-\beta$-D-glucopyranoside & 11 & \multirow{3}{*}{$\begin{array}{l}\text { El-Wakil and Eman , 2007; } \\
\quad \text { Miyase et al., 1996a }\end{array}$} \\
\hline & 3-O- $\beta$-D-glucopyranosyl-( $1 \rightarrow 3)$ - $\alpha$-L-arabinopyranosyl-quinovic acid-28- $O$ - $\beta$-D-glucopyranoside & 12 & \\
\hline & 3-O- $\beta$-D-glucopyranosyl- $(1 \rightarrow 3)-\alpha$-L-arabinopyranosyl-oleanolic acid & 13 & \\
\hline & 3-O- $\beta$-D-glucopyranosyl-( $(1 \rightarrow 3)-[\beta$-D-glucopyranosyl- $(1 \rightarrow 2)-] \alpha$-L-arabinopyranosyl-oleanolic acid & 15 & $\begin{array}{l}\text { El-Wakil and Eman, 2007; } \\
\quad \text { Miyase et al., 1996a }\end{array}$ \\
\hline & 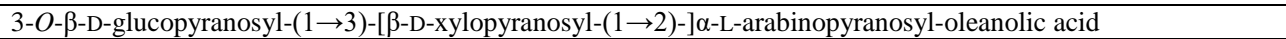 & 16 & \multirow{3}{*}{ Miyase et al., 1996a } \\
\hline & $\begin{array}{l}\text { 3- } O \text { - } \beta \text {-D-glucopyranosyl- }(1 \rightarrow 3) \text {-[ }[\beta \text {-D-glucopyranosyl- }(1 \rightarrow 2)-] \alpha \text {-L-arabinopyranosyl-oleanolic acid- } 28-O-\beta \text {-D- } \\
\text { glucopyranoside }\end{array}$ & 17 & \\
\hline & $\begin{array}{l}\text { 3- } O \text { - } \beta \text {-D-glucopyranosyl-( }(1 \rightarrow 3)-[\beta \text {-D-xylopyranosyl- }(1 \rightarrow 2)-] \alpha \text {-L-arabinopyranosyl-oleanolic acid-28- } O-\beta \text {-D- } \\
\text { glucopyranoside }\end{array}$ & 18 & \\
\hline & $\begin{array}{l}\text { 3-O- } \beta \text {-D-glucopyranosyl- }(1 \rightarrow 3)-[\beta \text {-D-xylopyranosyl- }(1 \rightarrow 2)-] \alpha \text {-L-arabinopyranosyl-27-hydroxyoleanolic acid-28- } \\
O \text { - } \beta \text {-D-glucopyranoside }\end{array}$ & 21 & El-Wakil and Eman, 2007 \\
\hline & 3-O-[2-O-sulfo- $\alpha$-L-arabinopyranosyl]-27-hydroxyursolic acid & 22 & \multirow{2}{*}{ Perrone et al., 2007} \\
\hline & 3-O-[2-O-sulfo- $\alpha$-L-arabinopyranosyl]-27-hydroxyursolic acid-28- $O$ - $\beta$-D-glucopyranoside & 23 & \\
\hline & $\begin{array}{l}\text { 3-O- } \beta \text {-D-glucopyranosyl-( } 1 \rightarrow 3) \text {-[ } \beta \text {-D-xylopyranosyl- }(1 \rightarrow 2) \text { - }] \alpha \text {-L-arabinopyranosyl-ursolic acid-28- } O \text { - } \beta \text {-D- } \\
\text { glucopyranoside }\end{array}$ & 28 & \multirow{2}{*}{ Miyase et al., 1996a } \\
\hline & $\begin{array}{l}\text { 3- } O \text { - } \beta \text {-D-glucopyranosyl- }(1 \rightarrow 3) \text {-[ } \beta \text {-D-xylopyranosyl- }(1 \rightarrow 2) \text { - }] \alpha \text {-L-arabinopyranosyl-27-hydroxyursolic acid-28- } \\
O \text { - } \beta \text {-D-glucopyranoside }\end{array}$ & 32 & \\
\hline & 3-O-sulfonyl-27-hydroxyoleanolic acid & 3 & \multirow{4}{*}{ Perrone et al., 2007} \\
\hline & 3- $O$-sulfonyl-27-hydroxyoleanolic acid-28- $O$ - $\beta$-D-glucopyranoside & 4 & \\
\hline & 3ß,23-disulfonyl-nahagenin & 8 & \\
\hline & 3,23-disulfate ester of3 $\beta, 23$-dihydroxyolean-13(18)-en-28-oic acid-28- $O$ - $\beta$-D-glucopyranoside & 9 & \\
\hline \multirow[t]{12}{*}{ F. cretica } & 3-O- $\beta$-D-glucopyranosyl-quinovicacid & 6 & Saleem et al., 2014 \\
\hline & 3-O- $\beta$-D-glucopyranosyl-hederagenin & 2 & Batterjee et al., 2002 \\
\hline & 3-O- $\beta$-D-glucopyranosyl-quinovic acid-28-O- $\beta$-D-glucopyranoside & 10 & Saleem et al., 2014 \\
\hline & 3-O- $\beta$-D-glucopyranosyl-( $1 \rightarrow 4)-\alpha$-L-rhamnopyranosyl-hederagenin & 33 & \multirow{2}{*}{ Batterjee et al., 2002} \\
\hline & 3-O- $\beta$-D-glucopyranosyl-( $(\rightarrow 5)-\alpha$-L-arabinofuranosyl- $(1 \rightarrow 4)$ - $\alpha$-L-rhamnopyranosyl-hederagenin & 34 & \\
\hline & 3-O- $\beta$-D-glucopyranosyl-( $(\rightarrow 2)$ - $\alpha$-L-arabinopyranosyl-hederagenin-28- $O$ - $\beta$-D-glucopyranoside & 35 & \multirow{3}{*}{ Abdel Khalik et al., 2000a } \\
\hline & $\begin{array}{l}\text { 3- } O \text { - } \beta \text {-D-glucopyranosyl-( }(1 \rightarrow 2)-\alpha \text {-L-arabinopyranosyl-oleanolic acid-28- } O \text {-[ } \beta \text {-D-glucopyranosyl- }(1 \rightarrow 6)-\beta \text {-D- } \\
\text { glucopyranosyl-] ester }\end{array}$ & 36 & \\
\hline & $\begin{array}{l}\text { 3- } O \text { - } \beta \text {-D-glucopyranosyl-( }(\rightarrow 2)-\alpha \text {-L-arabinopyranosyl-27-hydroxyoleanolic acid-28- } O \text {-[ } \beta \text {-D-glucopyranosyl- } \\
(1 \rightarrow 6)-\beta \text {-D-glucopyranosyl-]ester }\end{array}$ & 37 & \\
\hline & $\begin{array}{l}\text { 3- } O \text { - } \beta \text {-D-xylopyranosyl- }(1 \rightarrow 2)-\alpha \text {-L-arabinopyranosyl-27-hydroxyoleanolic acid-28- } O \text {-[ } \beta \text {-D-glucopyranosyl- } \\
(1 \rightarrow 6) \text { - } \beta \text {-D-glucopyranosyl-] ester }\end{array}$ & 38 & Abdel Khalik et al., 2000b \\
\hline & $\begin{array}{l}\text { 3- } O \text { - } \beta \text {-D-glucopyranosyl- }(1 \rightarrow 2)-\alpha \text {-L-arabinopyranosyl-olean-12-en-27-al-28-oic acid-28- } O \text {-[ } \beta \text {-D- } \\
\text { glucopyranosyl- }(1 \rightarrow 6)-\beta \text {-D-glucopyranosyl-] ester }\end{array}$ & 39 & Abdel Khalik et al., 2000a \\
\hline & $\begin{array}{l}\text { 3- } O \text { - } \beta \text {-D-xylopyranosyl- }(1 \rightarrow 2)-\alpha \text {-L-arabinopyranosyl- olean-12-en-27-al-28-oic acid-28- } O \text {-[ } \beta \text {-D-glucopyranosyl- } \\
(1 \rightarrow 6)-\beta \text {-D-glucopyranosyl-] ester }\end{array}$ & 40 & Abdel Khalik et al., 2000b \\
\hline & $3 \beta-O$-[(6-deoxy- $\alpha$-L-talopyranosyl)oxy]-22 $\alpha$-hydroxyurs-12-en-28-oic acid (fagonoside A) & 49 & Anjum et al., 2014 \\
\hline \multirow{6}{*}{$\begin{array}{l}\text { F. } \\
\text { glutinosa }\end{array}$} & 3-O- $\alpha$-L-arabinopyranosyl-ursolic acid-28-O- $\beta$-D-glucopyranoside & 24 & \multirow{6}{*}{ Melek et al., 2000} \\
\hline & 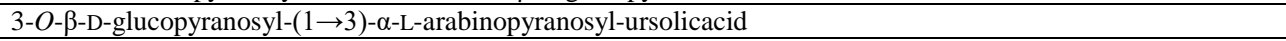 & 25 & \\
\hline & 3-O- $\beta$-D-glucopyranosyl-( $(\rightarrow 3)$-[ $\beta$-D-xylopyranosyl- $(1 \rightarrow 2)$ - $] \alpha$-L-arabinopyranosyl-ursolic acid & 27 & \\
\hline & $\begin{array}{l}\text { 3- } O \text { - } \beta \text {-D-glucopyranosyl- }(1 \rightarrow 3) \text {-[ } \beta \text {-D-glucopyranosyl- }(1 \rightarrow 2)-]-\alpha \text {-L-arabinopyranosyl-ursolic acid-28- } O \text { - } \beta \text {-D- } \\
\text { glucopyranoside }\end{array}$ & 29 & \\
\hline & $\begin{array}{l}\text { 3- } O \text { - } \beta \text {-D-glucopyranosyl- }(1 \rightarrow 3) \text {-[ } \beta \text {-D-glucopyranosyl- }(1 \rightarrow 2) \text { - }] \alpha \text {-L-arabinopyranosyl-27-hydroxyursolic acid-28- } \\
O \text { - } \beta \text {-D-glucopyranoside }\end{array}$ & 31 & \\
\hline & $\begin{array}{l}\text { 3- } O \text { - } \beta \text {-D-glucopyranosyl }(1 \rightarrow 3) \text { - }[\beta \text {-D-glucopyranosyl- }(1 \rightarrow 2)-] \alpha \text {-L-arabinopyranosyl-27-hydroxyoleanolic acid- } \\
28 \text { - } O \text { - } \beta \text {-D-glucopyranoside }\end{array}$ & 20 & \\
\hline \multirow[t]{13}{*}{ F. indica } & oleanolic acid-28-O-[ $\beta$-D-glucopyranosyl- $(1 \rightarrow 3)-\beta$-D-glucopyranosyl- $]$ ester & 41 & \multirow{2}{*}{ Shaker et al., 2000} \\
\hline & 3-O- $\alpha$-L-rhamnopyranosyl-quinovic acid-28- $O-\beta$-D-glucopyranoside & 42 & \\
\hline & 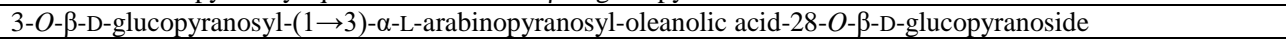 & 14 & \multirow{4}{*}{ Shaker et al., 2013} \\
\hline & 3-O- $\beta$-D-glucopyranosyl-( $(\rightarrow 3)-\alpha$-L-arabinopyranosyl-ursolic acid-28- $O$ - $\beta$-D-glucopyranoside & 26 & \\
\hline & $\begin{array}{l}\text { 3- } O \text { - } \alpha \text {-L-arabinopyranosyl- }(1 \rightarrow 3) \text {-[ } \beta \text {-D-glucopyranosyl- }(1 \rightarrow 2) \text { - }] \alpha \text {-L-arabinopyranosyl-oleanolic acid-28- } O \text { - } \beta \text {-D- } \\
\text { glucopyranoside (indicasaponin B) }\end{array}$ & 19 & \\
\hline & $\begin{array}{l}\text { 3-O- } \alpha \text {-L-arabinopyranosyl- }(1 \rightarrow 3) \text {-[ } \beta \text {-D-glucopyranosyl- }(1 \rightarrow 2)-] \alpha \text {-L-arabinopyranosyl-ursolic acid-28- } O \text { - } \beta \text {-D- } \\
\text { glucopyranoside } \quad \text { indicasaponin A) }\end{array}$ & 30 & \\
\hline & $\begin{array}{l}\text { 3- } O-\beta \text {-D-4- } O \text {-sulfonylglucopyranosyl- }(1 \rightarrow 3) \text { - } \alpha \text {-L-arabinopyranosyl-ursolic acid-28- } O \text { - } \beta \text {-D-glucopyranoside } \\
\text { (indicasaponin C) }\end{array}$ & 43 & \multirow{2}{*}{ Shaker et al., 1999} \\
\hline & $\begin{array}{l}\text { 3-O- } \beta \text {-D-4- } O \text {-sulfonylglucopyranosyl- }(1 \rightarrow 3) \text {-[ } \beta \text {-D-xylopyranosyl- }(1 \rightarrow 2)-] \alpha \text {-L-arabinopyranosyl-ursolic acid-28- } \\
O-\beta \text {-D-glucopyranoside (indicasaponin } \mathrm{D})\end{array}$ & 44 & \\
\hline & 3-oxo-12-en-23-O- $\beta$-D-glucopyranosyl-27-hydroxyolean-28-oic acid (indicacin) & 5 & Farheen et al., 2015 \\
\hline & $3 \beta, O$ - $\beta$-D-glucopyranosyl-23-hydroxytaraxer-20-en-28-oic acid & 45 & Farheen et al., 2015 \\
\hline & 23,28 -di- $O$ - $\beta$-D-glucopyranosyl-23-hydroxytaraxer-20-en-28-oic acid & 46 & $\begin{array}{l}\text { Farheen et al., 2015; Ansari } \\
\text { et al., } 1987\end{array}$ \\
\hline & 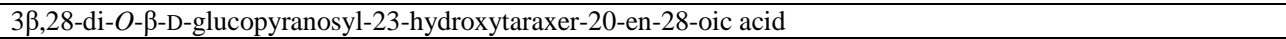 & 47 & Ansari et al., 1987 \\
\hline & $21,22 \alpha$-epoxy-23-O- $\beta$-D-glucopyranosyl-nahagenin & 48 & Ansari et al., 1988 \\
\hline F.mollis & 3-O- $\beta$-D-glucopyranosylquinovicacid & 6 & Sallam et al., 2014 \\
\hline
\end{tabular}




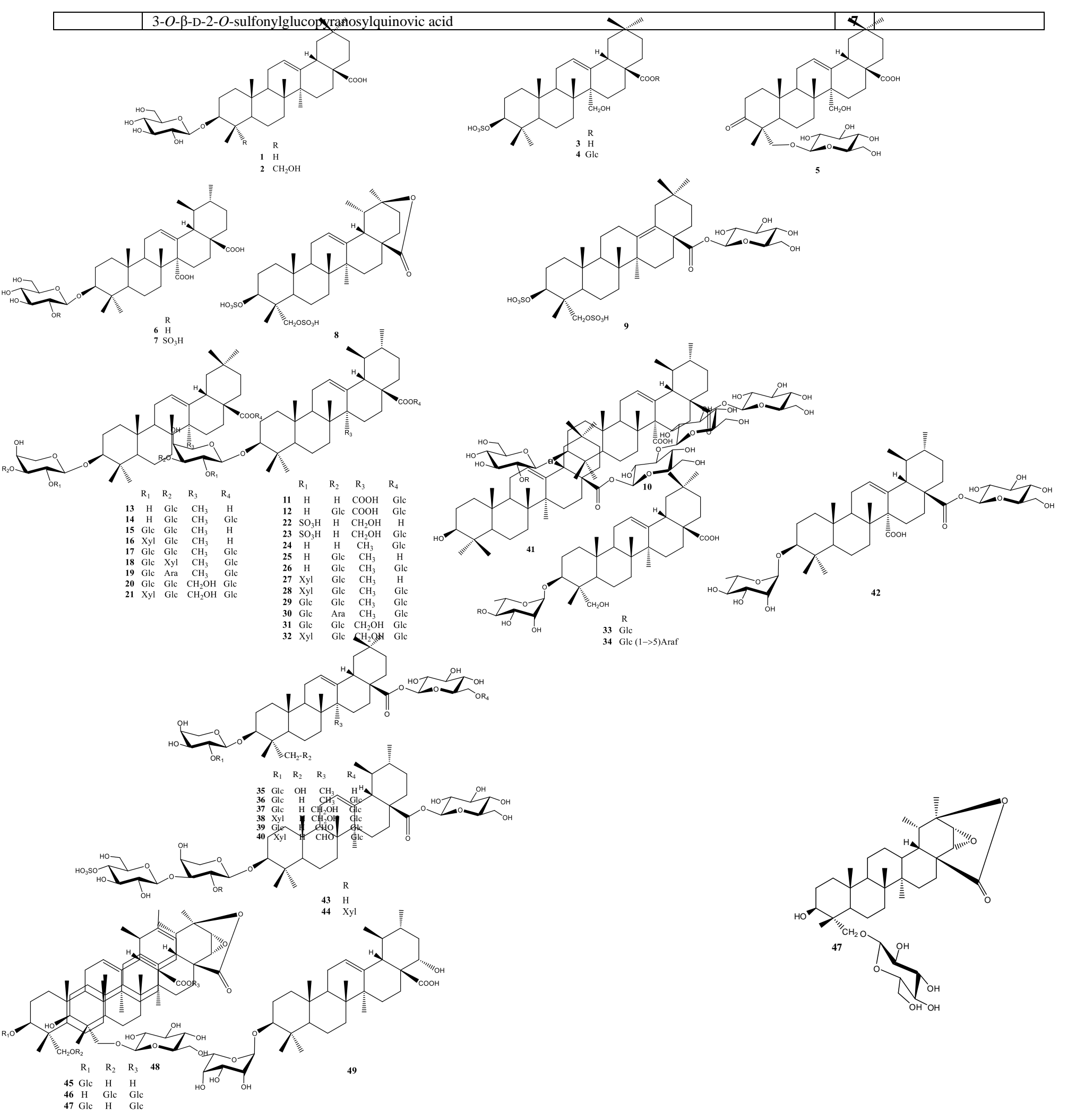

Fig.1: Structures of triterpenoid saponins isolated from Fagonia species 


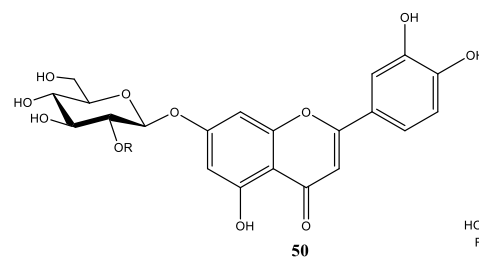

Fig.2 Structures of compounds $(\mathbf{2 6}, \mathbf{5 0}-$

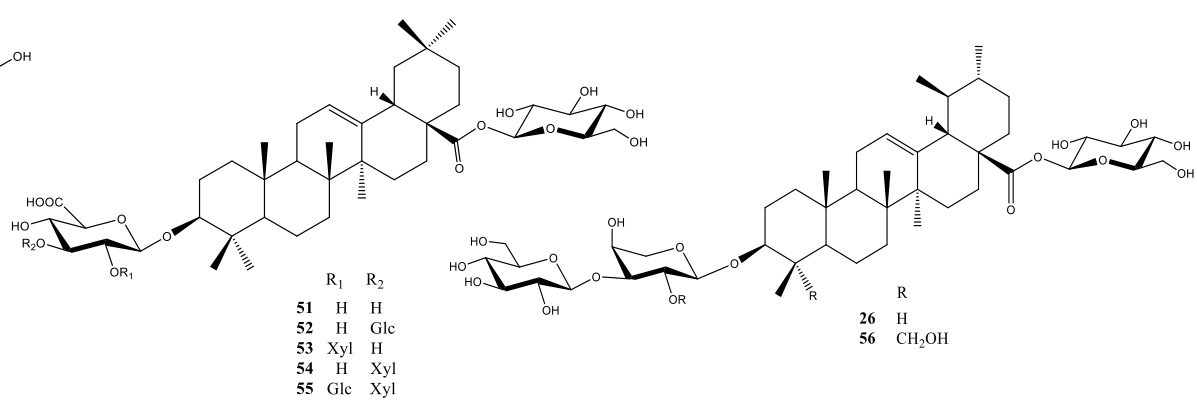

56) isolated from Fagonia

scabra 